\title{
Um estudo sobre os fatores de risco e proteção associados à violência sexual cometida contra adolescentes e jovens adultos
}

\author{
Simone dos Santos Paludo \\ Universidade Federal do Rio Grande \\ Eva Diniz Bensaja dei Schirò \\ Universidade Federal do Rio Grande do Sul
}

\begin{abstract}
Resumo
O estudo teve como objetivo identificar fatores de risco/proteção associados à ocorrência de violência sexual cometida contra adolescentes/jovens brasileiros, tanto no âmbito familiar como comunitário. Participaram do estudo 7316 jovens com idades entre 14 e 24 anos $(M=16,19 ; D P=1,82)$, sendo 3335 do sexo masculino. Os participantes provieram do estudo Juventude Brasileira. Do total da amostra, 6,6\% dos participantes revelaram serem vítimas de violência sexual no âmbito familiar e $6,0 \%$ no comunitário. O apoio familiar surgiu como uma variável de proteção, tanto para a violência na família quanto na comunidade. O ser menina e consumir álcool apareceram como variáveis de risco para ambos os domínios. Além disso, ter um familiar preso e alcoolismo na família foram variáveis identificadas como de risco no âmbito familiar, enquanto o ter passado fome, ter sofrido violência na comunidade e ter envolvimento com o tráfico foram as variáveis de risco associadas à violência sexual na comunidade.
\end{abstract}

Palavras-chave: risco; juventude; violência sexual.

\begin{abstract}
A study on the risk and protective factors associated with sexual violence against adolescents and youth. The aim of the study was to identify the risk and protection factors associated with sexual violence against Brazilian adolescents and emerging adults for both familiar and community contexts. The study counts with 7316 participants between the ages of $14-24$ years old $(M=16.19 ; S D=1.82)$ and 3974 were females from the data set "Brazilian Youth". From the whole sample $6.6 \%$ of the participants have suffered sexual violence within family and $6.0 \%$ within the community. Familiar support appears as a protective factor for sexual violence in family and in the community as well. Being a female and consume alcohol were the risk factors for both contexts. Besides, having a relative arrested, witnessed violence and alcoholism in family were the risk factors for sexual violence among the family, while starvation experiences, being victim of violence in community and involvement in drug trafficking were the risk factors for sexual violence in the community.

Keywords: risk; youth; sexual violence.
\end{abstract}

$\mathrm{E}$ stimar a prevalência de violência sexual contra crianças, adolescentes e jovens tem sido um desafio. Os números existentes ainda não conseguem traduzir com exatidão a real dimensão desse problema. Se por um lado o silêncio, o medo, a tolerância e a vergonha ainda são os principais obstáculos para a notificação dos casos, por outro os dados existentes acabam sendo restritos a serviços especializados, a fontes diversas ou a amostras de estudos que não representam a diversidade da população.

Esse cenário torna ainda mais complexa a identificação e a discussão dos fatores de risco e proteção inseridos nesse fenômeno. Embora a própria ocorrência de violência sexual por si só já determine a existência de riscos, é necessário conhecer a relação existente entre os fatores que incrementam o perigo e a vulnerabilidade das pessoas e aqueles que, de alguma forma, colaboram com a sua proteção.

De acordo com a World Health Organization (WHO), a violência sexual contra adolescentes é caraterizada pela ocorrência de qualquer tipo de atividade sexual, com alguém que esteja em um estágio psicossexual mais avançado, destinada à gratificação sexual dessa pessoa (WHO, 2002). Frequentemente, estas práticas sexuais são impostas à criança/adolescente, pela força, violência física e emocional, ou pela indução da sua vontade. A violência sexual pode ocorrer de diversas formas, envolvendo o contato físico (com ou sem penetração) e/ou a exposição a conteúdos eróticos/pornográficos (WHO, 2002).

A violência sexual pode ocorrer tanto ao nível intrafamiliar, como extrafamiliar. É denominado abuso sexual intrafamiliar quando ocorre no espaço doméstico e é praticado por pessoas afetivamente próximas à vítima. Já a violência sexual 
extrafamiliar tende a ser praticada fora do ambiente doméstico e, na maioria das vezes, é praticado por desconhecidos ou pessoas exteriores à família, como vizinhos, ou membros da comunidade (Koller \& De Antoni, 2004). É descrito que a violência sexual intrafamiliar tende a ser cometida em um período mais prolongado no tempo e tende a deixar mais sequelas na vítima, já que o agressor tende a ser uma pessoa de confiança, afetivamente próxima, com quem, na maioria das vezes, o convívio é mantido. Essa configuração faz com que haja uma maior dificuldade, por parte da vítima, para a revelação (Goodman-Brown, Edelstein, Goodman, Jones, \& Gordon, 2003).

De acordo com estimativas da OMS (Pinheiro, 2006), aproximadamente 150 milhões de meninas e 73 milhões de meninos com menos de 18 anos viveram algum tipo de violência sexual, apenas no ano de 2002. Diversos estudos revelam que as meninas têm três vezes mais chance de sofrer violência sexual que os meninos, em particular no espaço doméstico. Estudos realizados pela OMS em diversos países demonstram que 21\% das mulheres revelou ter sido vítima de violência sexual antes dos 15 anos, na maioria das vezes por um familiar próximo (Pinheiro, 2006).

No Brasil essa realidade não é diferente. Dados recentes publicados pelo Disque Direitos Humanos (SEDH, 2011), Disque Denúncia Nacional Módulo Criança e Adolescente, evidenciam o aumento de vítimas do sexo feminino quando as denúncias estão relacionadas à ocorrência de violência sexual. Segundo o último relatório publicado em 2011, as vítimas do sexo feminino são a maioria, chegando a $80 \%$ nas situações de exploração sexual. Os números preliminares divulgados pelo Sistema de Vigilância de Violências e Acidentes (VIVA, 2011) do Ministério da Saúde, também revelam que a violência sexual faz parte da vida de crianças e adolescentes. Dentre as 14.625 notificações de violência doméstica, sexual, física e outras agressões contra crianças menores de dez anos, a violência sexual contra crianças até os nove anos representa 35\% das notificações. Para aqueles com idades entre 10 e 14 anos, a violência sexual representa $10,5 \%$ das notificações e, para aqueles com idades entre 15 e 19 anos, o percentual de registros diminui para 5,2\%. O estudo mostra ainda que a maior parte das agressões aconteceu no âmbito doméstico $(64,5 \%)$.

Embora existam evidências importantes de que as violações ocorram com maior frequência no espaço privado e doméstico, é importante discutir os riscos existentes no espaço público. Nesse cenário, a comunidade surge também como um espaço propício à ocorrência da violência sexual, em particular quando a criança/ adolescente começa a frequentá-la de forma mais autônoma, como por exemplo, o caminhar para a escola. Esses percursos são identificados como aqueles em que tende a haver uma maior aproximação do perpetrador aos adolescentes. Frequentemente, o perpetrador é conhecido do adolescente, embora possa também não o ser (Pinheiro, 2006).

Sofrer algum tipo de violência sexual é algo que afeta um número considerável de pessoas ao redor do mundo, independentemente das condições socioeconômicas, de raça ou cultura. Contudo, alguns fatores psicossociais parecem estar relacionados positivamente ao aumento da vulnerabilidade dos jovens. Por exemplo, adolescentes do sexo feminino apresentam duas vezes mais probabilidade de relatar histórias de coerção sexual do que os adolescentes do sexo masculino (Foshee, Benefield, Ennett, Bauman, \& Suchindran, 2004; Howard, Wang, \& Yan, 2007; Irwin \& Rickert, 2005). O histórico de maus tratos e violência doméstica também tem sido destacado como um fator de risco importante (Hall-Smith, White, \& Holland, 2003). Outro fator que aparece com frequência associado à violência sexual é o consumo de álcool e drogas por parte da vítima (Champion et al., 2004), e por parte do autor da violência (Raghavan, Bogart, Elliott, Vestal, \& Schuster, 2004).

Tendo em vista que qualquer situação de vitimização oferece prejuízos para a vítima, tanto no desenvolvimento psicológico-emocional quanto social, a violência sexual passou a ser considerada um problema de Saúde Pública. No Brasil, a temática tem atraído a atenção de pesquisadores e tem sido incluída nas investigações de base populacional. Polanczyk, Zavaschi, Benetti, Zenker e Gammerman (2003) investigaram a exposição de 1193 jovens estudantes da oitava série do ensino fundamental das escolas estaduais da região metropolitana de Porto Alegre, através da aplicação do instrumento de "Triagem da Exposição de Crianças à Violência na Comunidade”. Os autores constataram que $2.3 \%$ da amostra estudada já haviam sido sexualmente violentadas, $4.5 \%$ já haviam testemunhado uma pessoa ser violentada e $27.9 \%$ relataram conhecer alguma vítima de violência sexual, evidenciando que jovens estão expostos a violações sexuais em seu cotidiano comunitário.

Em 2006, outro importante estudo avançou essa discussão e buscou identificar a prevalência de coerção sexual na vida de jovens. Moraes, Cabral e Heilborn (2006) ao entrevistar 4.634 jovens, com idades entre 18 e 24 anos, moradores de Porto Alegre, Rio de Janeiro e Salvador, verificaram que a prevalência da relação sexual forçada ultrapassava $10 \%$ nas três capitais. A análise dos dados revelou que essas ocorrências são mais frequentes entre as meninas com idades entre 10 e 19 anos (80,5\%), mas também ocorrem antes dos 10 anos, como identificado em $6,3 \%$ da amostra.

Esses dados reiteram a necessidade de estudos que ofereçam indicadores individuais, familiares e ambientais para a compreensão da violência sexual. Portanto, o objetivo do presente estudo visa identificar os fatores de risco/proteção associados à ocorrência de violência sexual cometida contra adolescentes e jovens adultos, tanto no nível familiar quanto na comunidade.

\section{Métodos}

\section{Delineamento}

O presente estudo faz parte de um estudo maior que teve como objetivo investigar as características biopsicossociais de adolescentes e jovens brasileiros (Pesquisa Nacional sobre Fatores de Risco e Proteção da Juventude Brasileira, de Koller, Cerqueira-Santos, Morais, \& Ribeiro, 2005). Foi utilizado um delineamento transversal com o objetivo de identificar os fatores de risco/proteção associados à violência sexual cometida contra 
adolescentes e jovens adultos.

\section{Participantes}

Participaram do estudo 7316 jovens com idades entre $14 \mathrm{e}$ 24 anos $(M=16,19 ; D P=1,82)$, sendo 3335 do sexo masculino e 3974 do sexo feminino. Todos os participantes foram contatados em instituições de atendimento e escolas da rede pública de sete capitais e três cidades brasileiras pertencentes a um nível sócioeconômico baixo, cujos critérios foram definidos pelo Instituto Brasileiro de Geografia e Estatística (IBGE, 2000). Os participantes foram selecionados a partir da base de dados do estudo Juventude Brasileira (Koller et al., 2005), composta por adolescentes e jovens oriundos de nove cidades, integrantes das cinco regiões do Brasil [Arcos (MG), Belo Horizonte (MG), Brasília (DF), Campo Grande (MS), Maués (AM), Porto Alegre (RS), Presidente Prudente (SP), Recife (PE), São Paulo (SP)].

\section{Procedimentos}

A pesquisa Juventude Brasileira (Koller et al., 2005) teve como principal objetivo caracterizar a população adolescente/ jovem de baixa renda do Brasil [(para informação sobre os critérios utilizados na seleção de participantes da pesquisa ver Cerqueira-Santos, Paludo, Schirò, \& Koller (2010)]. Os sujeitos foram convidados a participar da pesquisa em escolas da rede pública tanto municipais como estaduais. $\mathrm{O}$ questionário foi aplicado em grupo e preenchido individualmente.

As questões éticas referentes à pesquisa foram asseguradas quanto à integridade dos participantes, conforme consta da Resolução no 196/96, que regulamenta a pesquisa com seres humanos (Ministério da Saúde, 2002). Foi dada a garantia de sigilo das informações pessoais, assim como disponibilizada a assistência do grupo de pesquisa, caso algum participante necessitasse de apoio psicológico (Resoluções no 196/1996 do CNS). O projeto Pesquisa Nacional sobre Fatores de Risco e Proteção da Juventude Brasileira (Koller et al., 2005) foi aprovado pelo Comité de Ética de uma instituição de ensino superior, com o protocolo $n^{\circ} 2006 / 533$.

\section{Instrumento}

O questionário desenvolvido para a pesquisa Juventude Brasileira (Koller et al., 2005) foi utilizado como instrumento de pesquisa. O questionário original é composto por 109 questões, no qual se exploram diversos domínios da vida do(a) adolescente. $\mathrm{Na}$ sua generalidade, o questionário foi elaborado com base na literatura, com o objetivo de sistematizar informações que pudessem retratar a vida do(a) adolescente brasileiro. No presente estudo selecionaram-se as questões relacionadas ao objetivo, agrupadas em quatro dimensões principais: (1) informações sociodemográficas; (2) experiência de violência sexual; (3) consumo de substâncias; (4) medidas de autoestima, afeto positivo/negativo e apoio familiar; e (5) eventos de risco.

A experiência de violência sexual foi avaliada em uma escala Likert de cinco pontos ( 1 = nunca, 5 = muito frequente) a partir da resposta afirmativa a uma das três questões: (1) Tentou mexer no corpo, (2) Mexeu de fato no corpo, (3) Teve relação sexual forçada. A terceira dimensão reuniu informações sobre o uso lícito e ilícito de drogas. Para cada uma das substâncias listadas (ex., vinho, cerveja, cigarro, maconha, cheirar cola) o adolescente era inquirido sobre a sua experimentação (sim vs. não) de cada uma delas. No caso de se obter uma resposta afirmativa, inqueriase sobre o consumo no último mês, na última semana e no último ano. Essa medida foi adaptada de escalas utlizadas em enquetes com adolescentes (Carlini, Galduróz, Noto, \& Nappo, 2002; Galduróz, Noto, \& Carlini, 1997). A quarta dimensão compõe-se pela Escala de Auto-Estima (Rosenberg, 1979); versão reduzida da Escala de Afeto Positivo/Afeto Negativo validada para a populacao brasileira (Giacomoni, 2002); e Escala de Apoio Familiar, criada a partir de cinco afirmações sobre as relações familiares (ex. "Sinto-me seguro na minha familia", "Recebo a ajuda de que preciso da minha familia"). Cada uma dessas frases foi avaliada pelo adolescente em uma escala de três pontos (1 = discordo, 2 = não concordo nem discordo, 3 = concordo). Um escore final foi calculado a partir da média de cada uma das respostas, em que os valores mais altos correspondem a maior satisfação com o apoio familiar. A quinta dimensão foi formada por uma escala que mede diversas situações de risco (ex., envolvimento com tráfico de drogas, já ter vivido longe da família, ter um parente preso). A escala foi criada para a o estudo da Juventude Brasileira com base na literatura (Raffaelli, Koller, Cerqueira-Santos, \& Morais, 2007). As questões foram avaliadas em uma escala Likert de cinco pontos $(1=$ nunca, 5 $=$ muito frequente). Um escore final foi calculado a partir da média de cada uma das respostas, em que os valores mais altos correspondem a maior satisfação com o apoio familiar recebido.

A presença de elevada autoestima, afetos positivos e suporte familiar foram considerados os fatores protetores. Como fatores de risco considerou-se baixa autoestima, presença de afetos negativos e a vivência de eventos de risco.

Com o objetivo de identificar os fatores de risco/proteção associados à experiência de violência sexual foram definidos dois desfechos principais: (1) exposição à violência sexual na família; e (2) violência sexual na comunidade. Ambos foram apresentados como uma variável dicotômica ( $\operatorname{sim} /$ não) construída a partir da resposta afirmativa do jovem a uma das três questões: (1) Tentou mexer no corpo, (2) Mexeu de fato no corpo, (3) Teve relação sexual forçada.

Para tal transformação, inicialmente converteram-se as variáveis que, no questionário, apareciam em uma escala de Likert em variáveis dicotômicas, em que 1 correspondia à presença de violência sexual e 0 a sua ausência. Assim, à pontuação das respostas que variava de (1) nunca, (2) muito raramente, (3) raramente, (4) frequentemente, (5) muito frequentemente, procedeu-se o seguinte critério de conversão: nunca foi vítima de violência passou a ser o menor valor sendo o 1 transformado em 0 e quando havia qualquer resposta afirmativa de que o jovem já havia sido vítima de violência sexual pelo menos uma vez na vida os valores apontados na escala $2 / 3 / 4 / 5$ foram transformados em 1 .

\section{Análise dos dados}

De acordo com as duas variáveis dependentes (violência sexual na família e violência sexual na comunidade) foram criados dois modelos de regressão (Logit). Inicialmente, foram feitas correlações entre as variáveis dependentes e informações 
sociodemográficas, consumo de substâncias, medidas de autoestima, afeto positivo/negativo e apoio da família. As correlações identificadas como moderadas/fortes $(r>40, p<$ $0,05)$ foram incluídas no modelo de regressão.

\section{Resultados}

Da amostra geral, 44.3\% dos participantes revelaram já ter iniciado a sua vida sexual. A média de idade para a iniciação sexual foi 14.48 anos. No entanto, existe um percentual considerável ocorrendo antes dos 12 anos de idade (ver Tabela 1).

Relativamente à experiência de abuso sexual, os resultados

Tabela 1

Características da vida sexual da amostra de acordo com o sexo e a idade $(N=7316)$

\begin{tabular}{cc}
\hline Início da vida sexual & $n(\%)$ \\
\hline Iniciou vida sexual* & $3243(44,3)$ \\
Meninos & $1855(26,4)$ \\
Meninas & $1384(19,7)$ \\
$02-12$ anos & $329(11,1)$ \\
$13-18$ anos & $2601(87,7)$ \\
$19-24$ anos & $37(1,2)$ \\
\hline
\end{tabular}

* Idade média da iniciação sexual: 14,48 anos $( \pm 1,89)$.

obtidos revelam que, do total da amostra, $6,6 \%$ dos participantes revelaram ser vítimas de violência sexual no âmbito familiar e $6,0 \%$ na comunidade. Em ambas as situações as participantes do sexo feminino foram aquelas que mais apareceram como vítimas de violência sexual (4,2\% e 3,8\%, respectivamente; ver Tabela 2).

No modelo de regressão as variáveis independentes foram testadas separadamente para fatores de risco e de proteção associadas à ocorrência de violência sexual, tanto ao nível intrafamiliar como da comunidade. De acordo com a Tabela 3, verifica-se que apenas o apoio familiar $(\beta=-0,53, \mathrm{z}(5263)=4,23$, $p<0,001)$ se manteve no modelo como variável explicativa, evidenciando que o apoio familiar diminui em $41,3 \%$ a chance do adolescente/jovem sofrer algum tipo de violência sexual na sua família.

Os fatores de risco associados à violência sexual na família que se revelaram significativos no modelo foram: ser do sexo feminino aumenta em $36,4 \%$ a ocorrência da violência $(\beta=$ $0,31, \mathrm{z}(4896)=2,59, p<0,01)$, consumir bebida alcoólica $(\beta$ $=-0,32, \mathrm{z}(4896)=-2,73, p<0,001)$, presenciar situações de alcoolismo aumenta em $18,9 \%$ a chance de violação sexual no ambiente doméstico $(\beta=0,17, \mathrm{z}(4896)=2,50, p<0,01)$, vivenciar situações de violência em casa $(\beta=-0,97, \mathrm{z}(4896)=$ $-5,11, p<0,001)$, ter ou ter tido algum familiar preso $(\beta=-0,62$, $\mathrm{z}(4896)=-4,95, p<0,001)($ ver Tabela 4$)$.

Com o objetivo de identificar as variáveis associadas à vivência de violência sexual na comunidade foram utilizadas as mesmas variáveis independentes. Nesse modelo, constatou-se que a presença de afeto positivo $(\beta=-0,41, \mathrm{z}(5240)=2,51, p<$ $0,05)$ aparece como uma variável protetora contra a ocorrência de violência sexual na comunidade, assim como a presença de apoio familiar $(\beta=-0,55, \mathrm{z}(5240)=4,31, p<0,001)$, que mostrou

Tabela 2

Tipo de violência sexual sofrido por sexo, idade, orientação sexual e cor

\begin{tabular}{|c|c|c|}
\hline Perfil & $\begin{array}{c}\text { Já sofreu violência sexual } \\
\text { cometida por familiares } n(\%)\end{array}$ & $\begin{array}{c}\text { Já sofreu violência sexual } \\
\text { cometida pela comunidade } n(\%)\end{array}$ \\
\hline & $404(6,6)^{1}$ & $363(6,0)^{1}$ \\
\hline \multicolumn{3}{|l|}{ Idade } \\
\hline 14-18 anos & $374(6,1)$ & $334(5,5)$ \\
\hline $18-24$ anos & $30(0,5)$ & $29(0,5)$ \\
\hline \multicolumn{3}{|l|}{ Sexo } \\
\hline Feminino & $256(4,2)$ & $232(3,8)$ \\
\hline Masculino & $148(2,4)$ & $131(2,2)$ \\
\hline \multicolumn{3}{|l|}{ Orientação Sexual } \\
\hline Heterossexual & $362(6,3)$ & $325(5,8)$ \\
\hline Homossexual & $8(0,1)$ & $9(0,2)$ \\
\hline Bissexual & $7(0,1)$ & $4(0,1)$ \\
\hline Transexual & $2(0)$ & $1(0)$ \\
\hline \multicolumn{3}{|l|}{ Cor } \\
\hline Branca & $136(2,3)$ & $150(2,5)$ \\
\hline Negra & $68(1,1)$ & $48(0,8)$ \\
\hline Parda & $157(2,6)$ & $134(2,3)$ \\
\hline Amarela & $21(0,4)$ & $16(0,3)$ \\
\hline Indígena & $14(0,2)$ & $10(0,2)$ \\
\hline
\end{tabular}

${ }^{1}$ As categorias não são mutuamente exclusivas.

diminuir em $42,6 \%$ a chance do jovem de sofrer violência na comunidade.

Já as variáveis identificadas como de risco foram: ser do sexo feminino $(\beta=0,40, \mathrm{z}(4963)=1,72, p<0,001)$ aumenta a chance de ocorrência da violência em $50,4 \%$, ter passado fome $(\beta=-0,41, z(4963)=2,08, p<0,05)$, ter estado envolvido com o tráfico $(\beta=-0,57, \mathrm{z}(4963)=2,31, p<0,05)$, ter vivenciado violência na comunidade $(\beta=-0,65, \mathrm{z}(4963)=5,36, p<0,001)$ e já ter consumido alguma bebida alcóolica $(\beta=-0,71, z(4963)=$ $5,65, p<0,001$; ver Tabela 6).
Constatou-se que as variáveis associadas à ocorrência de violência sexual cometida contra adolescentes e jovens adultos se diferenciam de acordo com o contexto (familiar $v s$. comunitário) em que ocorre a violência. A percepção de apoio familiar foi a única variável que se revelou como protetora, face à violência sexual, em ambos os modelos, sugerindo que a qualidade das relações familiares toma especial importância na vida de crianças e jovens. Por outro lado, a identificação de fatores de risco evidenciou que a violência sexual tende a emergir em famílias que vivenciam situações de violação em seu cotidiano e em 
Tabela 3

Variáveis indepedentemente associadas com violência sexual intrafamiliar - fatores de proteção $(N=5268)$

\begin{tabular}{ll}
\multicolumn{1}{c}{ Variável } & \multicolumn{1}{c}{$\mathrm{B}$} \\
\hline Autoestima & $-0,06$ \\
Afeto positivo & $-0,06$ \\
Apoio familiar & $-0,53^{* * *}$ \\
Autoeficácia & $-0,15$ \\
Vinculação com a escola & $-0,02$ \\
\hline$* p<0,05 ; * * p<0,01 ; * * *<0,001$
\end{tabular}

$* p<0,05 ; * * p<0,01 ; * * * p<0,001$

Tabela 4

Variáveis indepedentemente associadas com violência sexual intrafamiliar - fatores de risco $(N=4904)$

\begin{tabular}{|c|c|}
\hline Variável & $\mathrm{B}$ \\
\hline Sexo & $0,31^{*}$ \\
\hline Afeto negativo & $-0,16$ \\
\hline Consumir bebida alcólica ${ }^{1,2}$ & $-0,32 * * *$ \\
\hline Consumir Cocaína ${ }^{1,2}$ & $-0,25$ \\
\hline Consumir Crack $^{1,2}$ & $-0,51$ \\
\hline Presenciar situações de alcoolismo & $0,17 * *$ \\
\hline Vivenciar violência em casa & $-0,97 * * *$ \\
\hline Ter familiar que está ou já esteve preso & $-0,62 * * *$ \\
\hline
\end{tabular}

${ }^{1}$ Resposta múltipla; ${ }^{2}$ Revelou ter usado alguma vez na vida; ${ }^{*} p<0,05$;

** $p<0,01 ; * * * p<0,001$

Tabela 5

Variáveis indepedentemente associadas com violência sexual na comunidade - fatores de proteção $(N=5245)$

\begin{tabular}{|c|c|}
\hline Variável & $\mathrm{B}$ \\
\hline Autoestima & 0,22 \\
\hline Afeto positivo & $-0,41^{*}$ \\
\hline Apoio familiar & $-0,55 * * *$ \\
\hline Autoeficácia & 0,11 \\
\hline Vinculação com a escola & $-0,11$ \\
\hline
\end{tabular}

$* p<0,05 ; * * p<0,01 ; * * *<<0,001$

Tabela 6

Variáveis indepedentemente associadas com violência sexual na comunidade - fatores de risco $(N=4971)$

\begin{tabular}{lc}
\hline \multicolumn{1}{c}{ Variável } & $\mathrm{B}$ \\
\hline Sexo & 0,40 \\
Afeto Negativo & $-0,17$ \\
Ter passado fome & $-0,41$ \\
Ter estado envolvido com o tráfico & $-0,57$ \\
Vivenciar violência na comunidade $^{1,2}$ & $-0,65$ \\
Consumir bebida alcólica $^{1,2}$ & $-0,71$ \\
Consumir Cocaína $^{1,2}$ & $-0,20$ \\
Consumir Crack $^{1,2}$ & 1,07 \\
\hline${ }^{1}$ Resposta múltipla; $^{2}$ Revelou ter usado alguma vez; $^{*} p<0,05 ; * * p$ & $* 0,01$; \\
&
\end{tabular}

comunidades em situação de precariedade social.

Neste estudo, do total de participantes, aproximadamente seis por cento revelaram já ter sofrido algum tipo de violência sexual. Esse é um valor ligeiramente inferior ao encontrado em outros estudos realizados no país (Moraes et al., 2006; Polanczyk et al., 2003; SEDH, 2011), embora revele que um número considerável de crianças/adolescentes se confronta, ao longo do seu desenvolvimento, com situações de violência e desrespeito dos seus direitos, tanto no seu ambiente familiar como na sua comunidade (Pinheiro, 2006). Também neste estudo, a prevalência de violência sexual cometida contra meninas foi superior a dos meninos, conforme relatado em pesquisas tanto nacionais (Moraes et al., 2006; Polanczyk et al., 2003), como internacionais (Foshee et al., 2004; Howard et al., 2007; Irwin \& Rickert, 2005; Pinheiro, 2006).

$\mathrm{O}$ apoio familiar sobressaiu como uma variável protetiva em ambos os contextos. Inúmeros estudos (Habzigang \& Caminha, 2004; Habigzang, Koller, Azevedo, \& Machado, 2005; Kellog 
\& Menard, 2003, Koller \& De Antoni, 2004) relatam que a violência sexual contra crianças/adolescentes tende a ocorrer em um contexto familiar de negligência ou desproteção, permeado de violência doméstica (Hall-Smith et al., 2003), em que predominam estilos parentais autoritários e relações de subordinação entre os membros familiares (Koller \& De Antoni, 2004). Sabe-se que muitas das situações de violência sexual ocorrem no espaço doméstico, sendo frequentemente praticadas por pessoas próximas à vítima (Pinheiro, 2006). Por isso, contextos familiares marcados pelo conflito e instabilidade são mais propícios para atitudes de desrespeito para com os elementos nele envolvidos, nomeadamente a violência sexual (Habzigang et al., 2005; Pinheiro, 2006). Os resultados obtidos no nosso estudo confirmam esta perspectiva e apontam para a importância das relações familiares saudáveis.

Constatou-se que o risco para a violência sexual cometida contra adolescentes/jovens adultos se associa à presença de alcoolismo, violência familiar e ter familiares que estão ou estiveram presos. No entanto, estas variáveis não devem ser interpretadas segundo uma perspectiva causal. Cowan, Cowan e Schulz (1996) já discutiam que fatores de risco relacionamse com eventos negativos que, quando presentes, poderiam aumentar a probabilidade de consequências negativas. Nesse sentido, é possível inferir que a combinação desses fatores pode representar um perigo. Portanto, quando a família não oferece o papel protetivo esperado, o desenvolvimento da criança/ adolescente tem um risco acrescido (Cohen \& Manarino, 2000; Jones et al., 2010).

O apoio familiar foi identificado como uma variável protetora para ambos os tipos de violência sexual. Ou seja, o apoio familiar minimiza não apenas o risco de violência sexual intrafamiliar, mas também extrafamiliar. Esse resultado demonstra que a combinação de fatores pode gerar proteção ou risco. Quando a família executa seu papel de cuidador e não apresenta situações de violação de direitos dentro do espaço doméstico diminui a possibilidade de ocorrência de violência sexual. Outra variável identificada como protetora face à violência sexual na comunidade foi a presença de afeto positivo. $\mathrm{O}$ afeto positivo, genericamente, caracteriza-se pelo humor, alegria, felicidade e satisfação com a vida. Possivelmente, os participantes com mais afeto positivo estiveram expostos a menos situações de tensão e violência e, por conseguinte, têm menor risco de se engajar em situações consideradas adversas ao desenvolvimento (Cohen \& Manarino, 2000; Habzigang et al., 2005; Jones et al., 2010).

Ainda na análise dos fatores de risco na comunidade foram identificadas as variáveis envolvimento com atividades de tráfico, ter sofrido violência na comunidade, ou ter passado fome. Constata-se que, neste caso, a presença de um fator de risco pode potencializar a exposição a outros riscos pela procura de alternativas de desenvolvimento, ou até mesmo de sobrevivência. Por exemplo, o adolescente/jovem pode envolverse com atividades consideradas marginais, com o intuito de obter proteção, como o envolvimento com a rede de tráfico. Por sua vez, o fornecimento dessa proteção pode colocar o adolescente/ jovem em uma situação vulnerável, sendo mais facilmente violentado nos seus direitos. O ter passado fome é outro exemplo desta dualidade risco/proteção. A necessidade de obter alimento pode conduzir ao envolvimento com atividades ilícitas, gerandose uma assimetria e, consequentemente, uma atitude de coerção sobre a vítima. É possível inferir que a fragilidade apresentada pela vítima nessas situações pode acabar expondo-a à violação sexual, uma vez que os autores de violência acabam por se aproveitar do poder existente nessa relação.

$\mathrm{O}$ fato de ser menina e de ter consumido bebida alcóolica surgiram como variáveis de risco para a violência sexual que ocorre tanto na família quanto na comunidade. A maior prevalência de violência sexual praticada contra as meninas tem sido identificada em outras pesquisas (Foshee et al., 2004; Habzigang et al., 2005; Howard et al.,2007; Irwin \& Rickert, 2005; Moraes et al.,2006; Pinheiro, 2006; Polanczyk et al., 2003). Por exemplo, um estudo de análise de registros de crianças e adolescentes (Kristensen, Oliveira, \& Flores, 1999; $N=1754$ ) vítimas de violência sexual, desenvolvido na região metropolitana de Porto Alegre, verificou que a maioria das vítimas tende a ser do sexo feminino. No entanto, Pereda, Guilera, Forns e GómezBenito (2009) ressaltam que desproporção de acordo com o sexo pode estar mais associada ao relato do abuso, nomeadamente pela identificação se a situação configura abuso, do que a real ocorrência de abuso. Tradicionalmente há uma preocupação com a violência sexual em meninas, enquanto os estereótipos de masculinidade levam a um sub-reconhecimento dos episódios de abuso sofridos por este sexo (Pereda et al., 2009). Além disso, segundo os autores, até há pouco tempo a própria definição de abuso excluía os rapazes.

O consumo de bebida alcóolica é descrito como outra variável associada à ocorrência de violência sexual, tanto para a vítima (Champion et al., 2004; Habzigang \& Caminha, 2004; Jones et al., 2010; Kellog \& Menard, 2003), quanto para o agressor (Raghavan et al., 2004). No presente estudo, não foi possível averiguar se o consumo de substâncias surgiu como uma consequência da violência sexual, ou a violência sexual ocorreu no contexto de consumo. No entanto, de acordo com Jones e colaboradores (2010) esse consumo estaria associado à violência sexual, em que o adolescente/jovem para se empoderar e se sentir autoconfiante recorreria ao consumo de bebidas alcóolicas.

Os resultados obtidos revelam a importância de entender o desenvolvimento, nomeadamente os seus fatores de risco e proteção, de uma forma integrada em que as variáveis se influenciam mutuamente. Ao investigar quais seriam os fatores de risco/proteção para a ocorrência de violência sexual contra adolescentes, tanto na família como na comunidade, verificouse que a violência sexual tende a associar-se à vivência de outros episódios de violência. $\mathrm{O}$ apoio familiar surgiu como um fator protetor para a violência sexual em ambos os contextos investigados. Outros estudos (Champion et al., 2004; Cohen \& Manarino, 2000; Habzigang et al., 2005; Jones et al., 2010; Raghavan et al., 2004) têm discutido que a instabilidade e violência familiar expõem os adolescentes/jovens a mais comportamentos considerados de risco, nomeadamente a participação em atividades infratoras e o consumo de substâncias. Nesse sentido, considera-se que a percepção de apoio familiar é um fator protetor face ao risco da violência sexual também na comunidade, já que adolescentes que provêm de famílias 
que avaliam como seguras e apoiantes têm menos tendência a envolver-se em comportamentos considerados desviantes.

Algumas limitações devem ser apresentadas ao presente estudo. A principal refere-se à falta de informação sobre a idade em que ocorreu a violência sexual, assim como informações sobre o autor da violência. Essa é uma limitação decorrente do questionário que pretendia caracterizar, genericamente, a juventude brasileira, e não a violência sexual em particular. Assim, dados que seriam importantes para uma melhor compreensão da situação não foram possíveis de obter. Não obstante, considera-se que os resultados são de extremo valor, já que nos permitiram uma caracterização genérica da situação de violência sexual cometida contra adolescentes/jovens no país. Ademais, os resultados permitiram aceder a uma contextualização das situações associadas à violência sexual. O objetivo do estudo não era encontrar relações causais, mas identificar fatores de risco e proteção associados à violência sexual. Conhecer indicadores individuais, familiares e ambientais é fundamental para a compreensão da violência sexual e para a organização de programas de prevenção realizados pelos serviços sociais, de saúde e nos trabalhos clínicos com esta população.

\section{Referências}

Carlini, E. A.; Galduróz, J. C.; Noto, A. R., \& Nappo, S. A. (2002). I Levantamento Domiciliar Sobre o Uso de Drogas Psicotrópicas no Brasil - 2001. São Paulo: CEBRID - Centro Brasileiro de Informações sobre Drogas Psicotrópicas.

Cerqueira-Santos, E., Paludo, S., Schirò, E., \& Koller, S. (2010). Gravidez na adolescência: análise contextual de risco e proteção. Psicologia em Estudo, 15(1), 72-85.

Champion, H., Foley, K., Durant, R., Hensberry, R., Altman, D., \& Wolfson, M. (2004). Adolescent sexual victimization, use of alcohol and other health risk behaviors. Journal of Adolescent Health, 35(4), 321-328.

Cohen, J. A., \& Mannarino, A. P. (2000). Predictors of treatment outcome in sexually abused children. Child Abuse \& Neglect, 24(7), 983-994.

Cowan, P. A., Cowan, C. P., \& Schulz, M. S. (1996). Thinking about risk and resilience in families. In E. M. Hetherington \& E. A. Blechman (Orgs.), Stress, coping, and resiliency in children and families (pp.1-38). Nova Jersey: Lawrence Erlbaum.

Foshee, V.A., Benefield, T.S., Ennett, S.T., Bauman, K.E., \& Suchindran, C. (2004). Longitudinal predictors of serious physical and sexual dating violence victimizacion during adolescence. Preventive Medicine, 39,1007-1016.

Galduróz, J., Noto, A. R., \& Carlini, E. A. (1997). IV Levantamento sobre o uso de drogas entre estudantes do $1^{\circ}$ e do $2^{\circ}$ graus em 10 capitais brasileiras. São Paulo: CEBRID.

Giacomoni, C. H. (2002). Bem-estar subjetivo infantil: conceito de felicidade e construção de instrumentos para avaliação. (Tese de Doutorado). Universidade Federal do Rio Grande do Sul, Porto Alegre. (000333187)

Goodman-Brown, T, B., Edelstein, R. S., Goodman, G. S., Jones, D. P. H., \& Gordon, D. S. (2003). Why children tell: a model of children's disclosure of sexual abuse. Child Abuse \& Neglect, 27, 525-540.

Habigzang, L. F., \& Caminha, R. M. (2004). Abuso sexual contra crianças e adolescentes: conceituação e intervenção clínica. São Paulo: Casa do Psicólogo.

Habigzang, L. F., Koller, S. H., Azevedo, G. A., \& Machado, P. X. (2005). Fatores de risco e de proteção na rede de atendimento a crianças e adolescentes vítimas de violência sexual. Psicologia Reflexão e Crítica, 21, 341-348.

Hall-Smith, P. H., White, J. W., \& Holland, L. J. (2003). A longitudinal perspective on dating violence among adolescent and college-age women. American Journal of Public Health, 93, 1104-1109.

Howard, D. E, Wang, M. Q., \& Yan, F. (2007). Psychosocial factors associated with reports of physical dating violence among U.S. adolescent females. Adolescence, 42, 311-324.

IBGE (2000). Censo 2000. Instituto Brasileiro de Geografia e Estatística. Recuperado de http://www.ibge..gov.br/censo

Irwin, C.E., \& Rickert, V.I. (2005). Coercive sexual experiences during adolescence and young adulthood: a public health problem. Journal of Adolescent Health, 36, 359-361.

Jones, D. J., Runyan, D. K., Lewis, T., Litrownik, A. J., Black, M. M., Wiley, T., ... Nagin, D. S. (2010). Trajectories of childhood sexual abuse and early adolescent HIV/AIDS Risk Behaviours: the role of other maltreatment, witnessed violence, and child gender. Journal of Clinical Child \& Adolescent Psychology, 39, 667-680.

Kellog, N. D., \& Menard, S. W. (2003). Violence among family members of children and adolescents evaluated for sexual abuse. Child Abuse \& Neglect, 27, 1367-1376.

Koller, S. H., Cerqueira-Santos, E., Morais, N. A., \& Ribeiro, J. (2005). Juventude brasileira. Relatório técnico. Washington DC: World Bank.

Koller, S. H., \& De Antoni, C. (2004). Violência intrafamiliar: Uma visão ecológica. In S. H. Koller (Org.), Ecologia do desenvolvimento humano: pesquisa e intervenção no Brasil (pp. 293-310). São Paulo: Casa do Psicólogo.

Kristensen, C. H., Oliveira, M. S., \& Flores, R. Z. (1999). Violência contra crianças e adolescentes na grande Porto Alegre - Parte B: pode piorar? In Associação de Apoio à Criança e ao Adolescente - AMENCAR (Org.), Violência doméstica (pp. 104-117). Brasília: UNICEF.

Ministério da Saúde (2002). Notificação de maus-tratos contra crianças e adolescentes pelos profissionais de saúde: um passo a mais na cidadania em saúde. Brasília: Secretaria de Assistência à Saúde.

Moraes, C. L., Cabral, C. S., \& Heilborn, M. L. (2006). Magnitude e caracterização de situações de coerção sexual vivenciadas por jovens de três grandes capitais brasileiras: Porto Alegre, Rio de Janeiro e Salvador. Cadernos de Saúde Pública, 22(7), 1493-1504.

Pereda, N., Guilera, G., Forns, M., \& Gómez-Benito, J. (2009). The prevalence of child sexual abuse in community and student samples: a meta-analysis. Clinical Psychology Review, 29, 328-338.

Pinheiro, P. S. (2006). World report on violence against children. National Criminal Justice Reference Service. Recuperado de http://www.ncjrs.gov/ App/Publications/abstract.aspx?ID=239950

Polanczyk, G. V., Zavaschi, M. L., Benetti, S., Zenker, R., \& Gammerman, P. W. (2003). Violência sexual e sua prevalência em adolescentes de Porto Alegre, Brasil. Revista de Saúde Pública, 37(1), 8-14.

Raghavan, R., Bogart, L. M., Elliott, M. N., Vestal, K. D., Schuster, M. A. (2004). Sexual victimization among a national probability sample of adolescent women. Perspectives on Sex and Reproductive Health, 36(6), 225-232.

Raffaelli, M., Koller, S. H., Cerqueira-Santos, E., \& de Morais, N. A. (2007). Developmental risks and psychosocial adjustment among low-income Brazilian youth. Development and Psychopathology, 19, 565-584.

Rosenberg, M. (1979). Conceiving the self. Nova Iorque: Basic Books.

Secretaria Especial dos Direitos Humanos. (2011). Relatório Disque Direitos Humanos, Módulo Criança e Adolescente. Recuperado de http://portal. mj.gov.br/sedh/spdca/T/RELATORIO\%202011\%20_agosto_.pdf

VIVA (2011). Sistema de Vigilância de Violências e Acidentes do Ministério da Saúde. Dados Preliminares. Recuperado de http://portalsaude.saude.gov.br/ portalsaude/noticia/5242/162/abuso-sexual-e-o-segundo\%3Cbr\%3E-maiortipo-de-violencia.html.

World Health Organization (2002). World Report on Violence and Health Geneva: WHO. 
404 S. S. Paludo \& E. D. B. Schirò

Simone dos Santos Paludo, doutora em Psicologia pela Universidade Federal do Rio Grande do Sul, é professora adjunta da Universidade Federal do Rio Grande. Endereço para correspondência: Universidade Federal do Rio Grande, Departamento de Educação e Ciências do Comportamento. Av. Itália, km 8, 96201900 - Rio Grande, RS. E-mail: paludo.simone@gmail.com.

Eva Diniz Bensaja dei Schirò é doutoranda de Psicologia pela Universidade Federal do Rio Grande do Sul. E-mail: evadiniz@gmail.com 